\title{
A WIND POWER PREDICTION METHOD BASED ON BAYESIAN FUSION
}

\author{
Jianqi $\mathrm{An}^{1,2,3}$, Zhangbing Chen ${ }^{1,2}$, Min $\mathrm{Wu}^{1,2}$, Takao Terano ${ }^{3}$, \\ Min Ding ${ }^{1,2}$, and Hua Xie ${ }^{1,2}$ \\ ${ }^{1}$ School of Automation, China University of Geosciences, \\ Wuhan, 430074, China \\ ${ }^{2}$ Hubei key Laboratory of Advanced Control and Intelligent Automation for \\ Complex Systems, Wuhan, 430074, China \\ ${ }^{3}$ Department of Computer Science, School of Computing, Tokyo Institute of \\ Technology, Yokohama, 226-8502, Japan
}

\begin{abstract}
Wind power prediction (WPP) is of great importance to the safety of the power grid and the effectiveness of power generations dispatching. However, the accuracy of WPP obtained by single numerical weather prediction (NWP) is difficult to satisfy the demands of the power system. In this research, we proposed a WPP method based on Bayesian fusion and multisource NWPs. First, the statistic characteristics of the forecasted wind speed of each-source NWP was analysed, pre-processed and transformed. Then, a fusion method based on Bayesian method was designed to forecast the wind speed by using the multi-source NWPs, which is more accurate than any original forecasted wind speed of each-source NWP. Finally, the neural network method was employed to predict the wind power with the wind speed forecasted by Bayesian method. The experimental results demonstrate that the accuracy of the forecasted wind speed and wind power prediction is improved significantly.
\end{abstract}

\section{KEYWORDS}

Wind Power Prediction, Numerical Weather Prediction, Bayesian Fusion, Wind Speed Prediction

\section{INTRODUCTION}

Wind power is a renewable, clean, plentiful and widely distributed energy, and does not produce greenhouse gas during operation [1-3]. WPP is of great importance to the safety of the power system and the effectiveness of power generations dispatching, which has become one of the most attractive research fields [4-6]. As wind power is major determined by the wind speed, the most key factor to predict accurate wind power is to forecast accurate wind speed. However, the wind speed is easily affected by many factors, such as air pressure, cloud chart, temperature, etc. These factors make the wind speed fluctuant and hard to be forecasted [7]. Therefore, the accurate forecasting of the wind speed is of great significance to achieve the effective prediction of wind power.

There are two main types of WPP approaches: physical approaches and statistical approaches. The physical approaches means that the wind power is predicted by the mathematical model of the generation process which is very difficult to build and need many hard-measured parameters.

David C. Wyld et al. (Eds) : ITCS, SIP, CST, ARIA, NLP - 2017

pp. 27- 37, 2017. @ CS \& IT-CSCP 2017

DOI : 10.5121/csit.2017.70303 
The statistical approaches to predict wind power use the weather forecast, combining with the operating condition, to obtain the value of the wind power based on the historical values relationship between the power and NWP. WPP is considered at different time scales: long-term prediction (several days or weeks, even mouths ahead), short-term prediction (1-2 days ahead) and very short-term prediction (several minutes to hours ahead). The difficulty of short-term prediction of wind power is much more than that of the very short-term prediction; while the required accuracy of short-term prediction is much higher than that of the long-term prediction.

Many literatures have demonstrated some good results about the short-term wind power prediction. In [8], a wind-forecasting method based on intrinsic time-scale decomposition (ITD) and least squares support vector machine (LS-SVM) was proposed. In [9], a time series model of WPP at different time scales was studied. In this study, with the step of the prediction increasing, the error would rise up, since the time series model used its own previous forecast value. In [10], a wind power prediction model based on empirical mode (EMD) and support vector machine (SVM) was proposed to reduce the influence of non-linear and non-stationary characteristics on wind power prediction. In [11], GUO Pengfei et al. presented a short-term wind power prediction method based on genetic algorithm to optimize RBF neural network. The RBF neural network was optimized by genetic algorithm to get the best value of the weights, base width and centre of the basis function of RBF neural network. In [12], a method based on sliding window weighted recursive least squares for wind power prediction was proposed. The historical data in the method was weighted so that the model would be able to adapt the varied environment. In [13], the constraint factor particle swarm optimization was used to optimize the parameters of the autoregressive model to accurately predict the wind power.

Overall, scholars have proposed many methods for WPP, but most of the researches only use the single NWP data to predict the wind power. The NWP data cannot always accurately predict the actual weather, so using only one NWP can hardly ensure the accuracy of wind power prediction. In this work, a new WPP method based on Bayesian method and multi-source NWPs was proposed. In this method, three independent NWPs given by different weather forecast companies, called multi-source NWPs, are employed and fused using Bayesian method to predict wind power. The three independent NWPs have different characteristics; each one has its own advantages and disadvantages. This fusion method can fully use the complementarity of the three NWPs, which can effectively improve the statistical accuracy of WPP.

\section{PROBLEM DESCRIPTION AND SCHEME OF WPP}

It is generally believed that the power of wind generation is directly proportional to the wind speed. In the meantime, the temperature, humidity, pressure and other weather factors have a certain impact on the power of wind generation. But based on our research, in some wind farms, the impact on the wind power caused by these factors is limited. We established a relational model between the actual measured wind speed and the actual wind power by using a neural network. The accuracy of the model is over $95 \%$, which means that the wind power can be predicted by introducing the wind speed as a single input. Therefore, the key problem of WPP for this type of wind farm is how to obtain an accurate forecasting wind speed.

In the research, there are three independent NWPs provided by different weather forecast companies. The problem we need to solve is to predict the wind power generation of the wind farm at intervals of 15 minutes from 00:00 to 24:00 of the next day according to the multi-sources NWPs. Thus there are 96 output prediction points which is used for dispatching power generations by power grid companies. 
In this research, first, a relational model between wind speed and wind power was established based on a back propagation neural network (BPNN) which was trained by the historical data of actual measured wind speed and actual wind power. By using the data driven method, the inherent relationship of the wind power generation is described. Then, instead of using one NWP, a Bayesian method was designed to fuse the three independent NWPs to calculate the wind speed which is more accurate than that of any of the three NWP. Finally, the wind speed forecasted by the Bayesian method is imported into the relational model to predict the wind power.

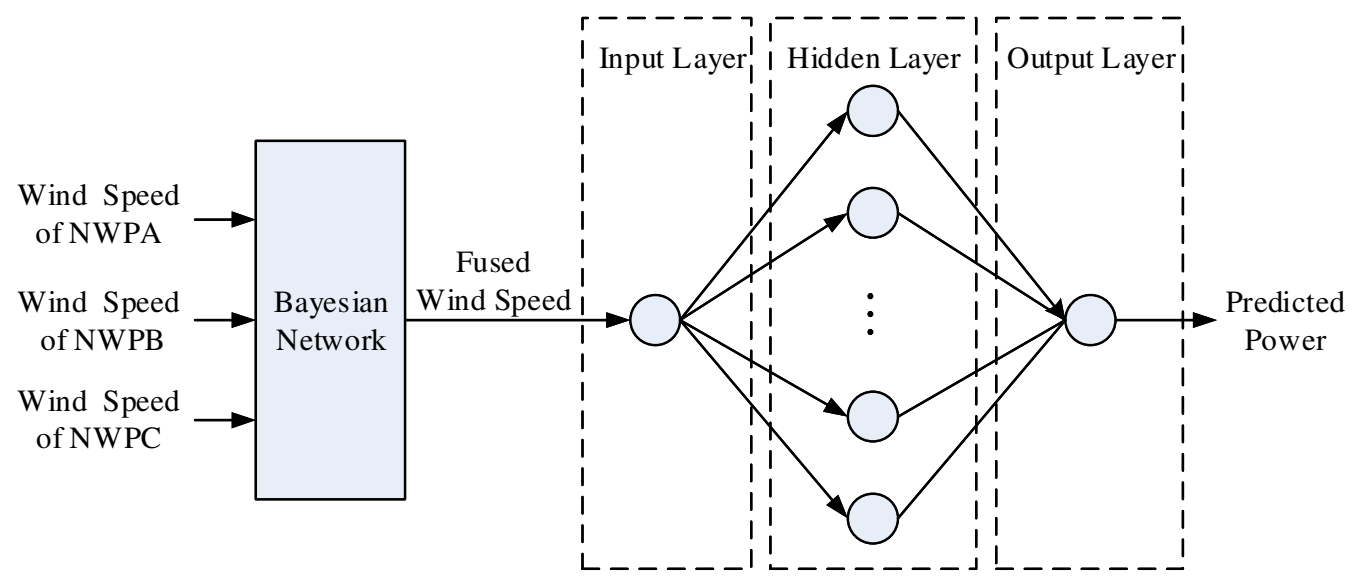

Figure 1. The scheme diagram of the proposed WPP method

In this paper, three layers BPNN structure is applied to build the relational model between wind speed and wind power, which contains one input node which is the wind speed and one output node which is the wind power. The incentive functions of the model are logsig and purelin, respectively. Since the basic idea of the BPNN is clear, this paper will not discuss further.

\section{ANALYSIS OF WIND SPEEd CHARACTERISTICS OF INDEPENDENT NWPS}

Three NWPs, defined as NWPA, NWPB and NWPC, have different characteristics. In the view of the instantaneous characteristics, sometimes NWPA is more accurate; sometimes NWPB and/or NWPC are/is more accurate. In the view of statistical characteristics, the wind speed values of the three NWPs and the actual measured wind speed value basically follow the normal distribution. The statistical characteristics of the historical actual wind speed and the wind speed of historical three NWPs are shown in Figure 2, which illustrates that the means and variances of each wind speed value are different. The values of the means and variances of each wind speed are shown in the Table 1.

Figure 3 (a), (b) and (c) show the comparison of forecasting wind speed of each NWP and the actual measured speed value in a certain period of history. As is shown the figure, the errors between the forecasting wind speed and the actual speed are fluctuating. The three kinds of wind speed of the NWPs fluctuate around a certain value, which is shown in Figure 4. Figure 4 (a), (b) and (c) show the fluctuation of the wind speed of NWPA, NWPB and NWPC when the actual measured wind speed is between $6 \mathrm{~m} / \mathrm{s}$ and $6.5 \mathrm{~m} / \mathrm{s}$. As mentioned above, sometimes NWPA is more accurate; sometimes NWPB and/or NWPC are/is more accurate. Therefore, a suitable method should be considered to fuse the three forecasting wind speed in order to calculate a more accurate wind speed. 
Table.1 Mean value and variance of each wind speed

\begin{tabular}{|l|l|l|l|l|}
\hline & Actual wind speed & $\begin{array}{l}\text { Wind speed of } \\
\text { NWPA }\end{array}$ & $\begin{array}{l}\text { Wind speed of } \\
\text { NWPB }\end{array}$ & $\begin{array}{l}\text { Wind speed of } \\
\text { NWPC }\end{array}$ \\
\hline Mean value & 7.78 & 7.84 & 9.01 & 6.73 \\
\hline Variance & 10.09 & 13.96 & 11.93 & 6.49 \\
\hline
\end{tabular}

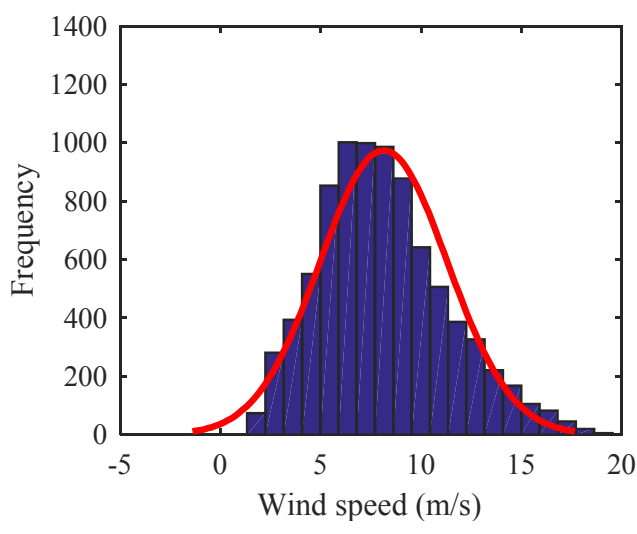

(a) Distribution of actual wind speed

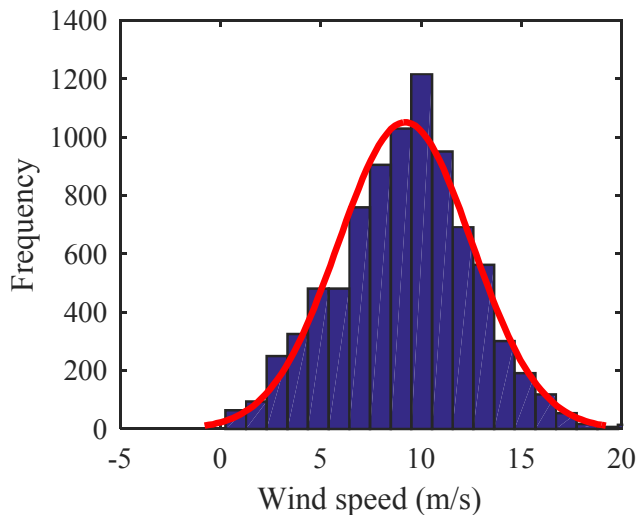

(c) Distribution of wind speed of NWPB

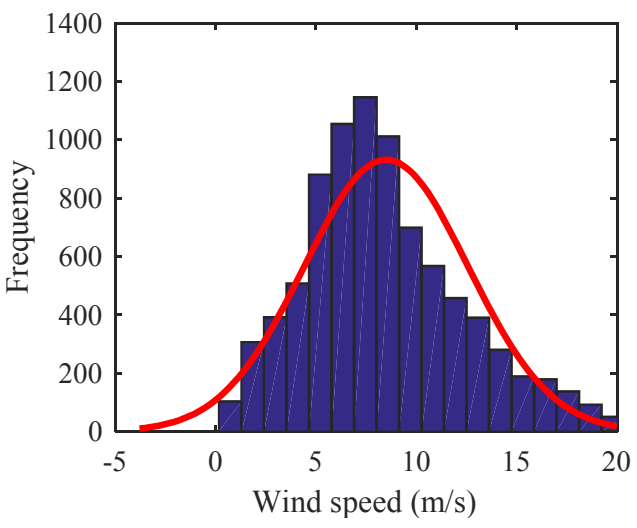

(b) Distribution of wind speed of NWPA

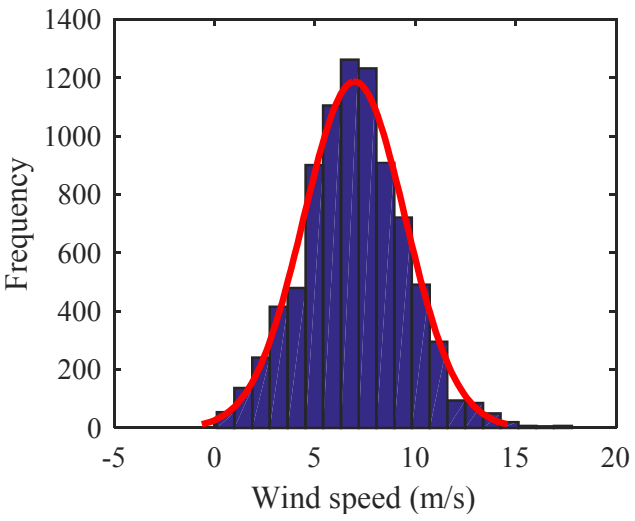

(d) Distribution of wind speed of NWPC

Figure 2. Distribution of each wind speed

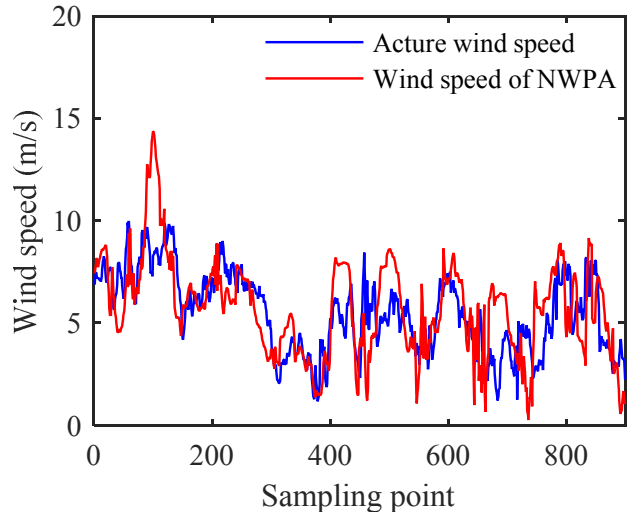

(a) Actual wind speed and wind speed of NWPA

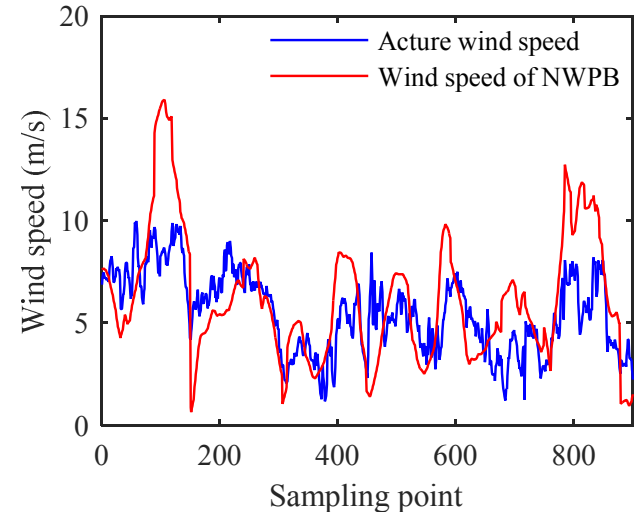

(b) Actual wind speed and wind speed of NWPB 


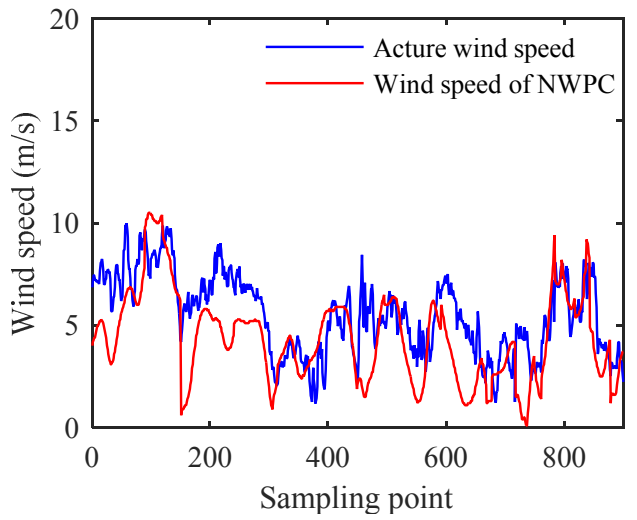

(c) Actual wind speed and wind speed of NWPC

Figure 3. The relationship between actual wind speed and wind speed of each NWP

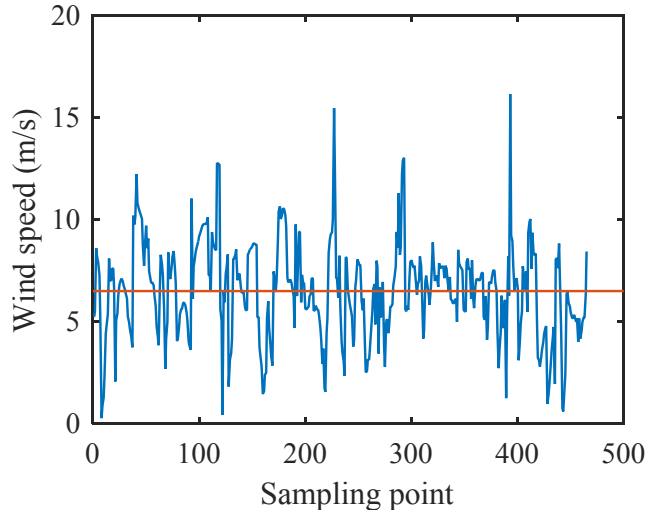

(a) Fluctuation of wind speed of NWPA

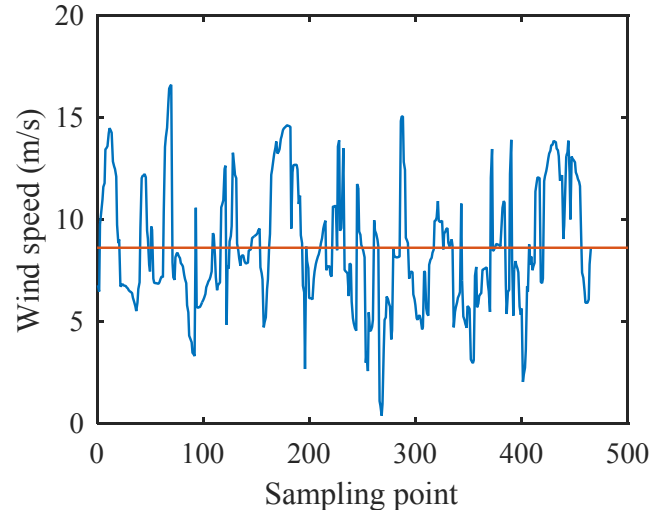

(b) Fluctuation of wind speed of NWPB

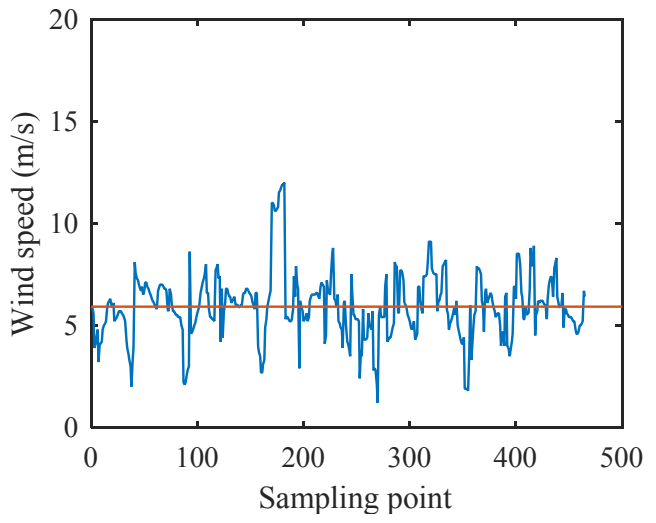

(c) Fluctuation of wind speed of NWPA

Figure 4. Fluctuation of the forecasting wind speed of each NWP when the actual wind speed is between $6 \mathrm{~m} / \mathrm{s}$ and $6.5 \mathrm{~m} / \mathrm{s}$. 


\section{BAYESIAN METHOd FOR FUSING THE MULTI-SOURCE NWPS}

In the view of the statistical characteristics, the wind speed values of three NWPs basically meet the normal distribution, but their feature are distinct account of different means and variance. It is possible to achieve a more accurate forecasting wind speed by fusing these three original forecasting wind speed values of the NWPs. The fused wind speed is more effective to predict the wind power.

In this paper, the three wind speed values of NWPs, defined as $x_{A}, x_{B}$ and $x_{C}$, are fused by using Bayesian method. Since the means of the history data of $x_{A}, x_{B}$ and $x_{C}$ are not equal, we make a transformation to let the means of them be equal in order to facilitate the solution. The transformed wind speeds are defined as $x_{A}^{\prime}, x_{B}^{\prime}$ and $x_{C}^{\prime}$. The transformation method is shown in equation (1),

$$
\begin{aligned}
& x_{A}^{\prime}=x_{A}+\left(u_{r}-u_{A}\right), \\
& x_{B}^{\prime}=x_{B}+\left(u_{r}-u_{B}\right), \text { and } \\
& x_{C}^{\prime}=x_{C}+\left(u_{r}-u_{C}\right),
\end{aligned}
$$

where $u_{r}$ denotes the mean value of the actual wind speed; $u_{A}, u_{B}$, and $u_{C}$ denote the mean values of wind speed of NWPA, NWPB, and NWPC. After the transformation, the mean values of the three wind speed of NWPs are equal. Then adopting the Bayesian method, the transformed wind speed values are fused into one.

The fused wind speed is defined as $x$. The Bayesian fusion equation is given by

$$
p\left(x \mid x_{A}^{\prime}, x_{B}^{\prime}, x_{C}^{\prime}\right)=\frac{p\left(x_{A}^{\prime}, x_{B}^{\prime}, x_{C}^{\prime} \mid x\right) p(x)}{p\left(x_{A}^{\prime}, x_{B}^{\prime}, x_{C}^{\prime}\right)}=\frac{p\left(x_{A}^{\prime} \mid x\right) p\left(x_{B}^{\prime} \mid x\right) p\left(x_{C}^{\prime} \mid x\right) p(x)}{p\left(x_{A}^{\prime}, x_{B}^{\prime}, x_{C}^{\prime}\right)}
$$

where $p(x)$ is the probability of the wind speed $x ; p\left(x_{A}^{\prime}, x_{B}^{\prime}, x_{C}^{\prime} \mid x\right)$ is the probability of forecast wind speed values of $x_{A}^{\prime}, x_{B}^{\prime}$, and $x_{C}^{\prime}$ when the wind speed is $x$; is the $p\left(x \mid x_{A}^{\prime}, x_{B}^{\prime}, x_{C}^{\prime}\right)$ is the probability of the wind speed $x$ when the forecast wind speed values of NWPA, NWPB, and NWPC are $x_{A}^{\prime}, x_{B}^{\prime}$ and $x_{C}^{\prime}$, respectively. $p(x)$ and $p\left(x_{A}^{\prime}, x_{B}^{\prime}, x_{C}^{\prime} \mid x\right)$ are priori probabilities which can be calculated by the historical data. $p\left(x \mid x_{A}^{\prime}, x_{B}^{\prime}, x_{C}^{\prime}\right)$ is the posteriori probability of the wind speed $x$. In this method, the purpose is to find the $x$ whose posteriori probability $p\left(x \mid x_{A}^{\prime}, x_{B}^{\prime}, x_{C}^{\prime}\right)$ is the highest.

The wind speed meet normal distribution, they are described as

$$
\begin{aligned}
& x \sim N\left(x_{0}, \sigma_{0}^{2}\right), \\
& x_{A}^{\prime} \sim N\left(u_{r}, \sigma_{A}^{2}\right), \\
& x_{B}^{\prime} \sim N\left(u_{r}, \sigma_{B}^{2}\right), \text { and } \\
& x_{C}^{\prime} \sim N\left(u_{r}, \sigma_{C}^{2}\right) .
\end{aligned}
$$

Let $a=\frac{1}{p\left(x_{A}^{\prime}, x_{B}^{\prime}, x_{C}^{\prime}\right)}$ which is a constant. The equation (2) can be transformed to 


$$
\begin{aligned}
p\left(x \mid x_{A}^{\prime}, x_{B}^{\prime}, x_{C}^{\prime}\right)= & \frac{1}{\sqrt{2 \pi \sigma_{A}}} \exp \left\{-\frac{1}{2}\left[\frac{x_{A}^{\prime}-u_{r}}{\sigma_{A}}\right]^{2}\right\} \times \frac{1}{\sqrt{2 \pi \sigma_{B}}} \exp \left\{-\frac{1}{2}\left[\frac{x_{B}^{\prime}-u_{r}}{\sigma_{B}}\right]^{2}\right\} \\
& \times \frac{1}{\sqrt{2 \pi \sigma_{C}}} \exp \left\{-\frac{1}{2}\left[\frac{x_{C}^{\prime}-u_{r}}{\sigma_{C}}\right]^{2}\right\} \times \frac{1}{\sqrt{2 \pi \sigma_{0}}} \exp \left\{-\frac{1}{2}\left[\frac{r-r_{0}}{\sigma_{0}}\right]^{2}\right\} \\
= & \exp \left\{-\frac{1}{2}\left[\frac{x_{A}^{\prime}-u_{r}}{\sigma_{A}}\right]^{2}-\frac{1}{2}\left[\frac{x_{B}^{\prime}-u_{r}}{\sigma_{B}}\right]^{2}-\frac{1}{2}\left[\frac{x_{C}^{\prime}-u_{r}}{\sigma_{C}}\right]^{2}-\frac{1}{2}\left[\frac{x-x_{0}}{\sigma_{0}}\right]^{2}\right\} \\
& \times \frac{1}{\sqrt{2 \pi \sigma_{A}}} \times \frac{1}{\sqrt{2 \pi \sigma_{B}}} \times \frac{1}{\sqrt{2 \pi \sigma_{C}}} \times \frac{1}{\sqrt{2 \pi \sigma_{0}}}
\end{aligned}
$$

where $\sigma_{A}, \sigma_{B}$, and $\sigma_{C}$ denote the root mean square of $x_{A}^{\prime}, x_{B}^{\prime}$ and $x_{C}^{\prime}$, respectively.

The exponential part in the above equation is a quadratic function with respect to $x$, so $p\left(x \mid x_{A}^{\prime}, x_{B}^{\prime}, x_{C}^{\prime}\right)$ is still normal distribution. It can be described as $N\left(x_{N}, \sigma_{N}^{2}\right)$. Then

$$
p\left(x \mid x_{A}^{\prime}, x_{B}^{\prime}, x_{C}^{\prime}\right)=\frac{1}{\sqrt{2 \pi \sigma_{N}}} \exp \left\{-\frac{1}{2}\left[\frac{x-x_{N}}{\sigma_{N}}\right]^{2}\right\} .
$$

$x_{N}$ can be obtained by comparing the parameters between the equation (4) and (5),

$$
x_{N}=\left[\frac{x_{A}^{\prime}}{\sigma_{A}^{2}}+\frac{x_{B}^{\prime}}{\sigma_{B}^{2}}+\frac{x_{C}^{\prime}}{\sigma_{C}^{2}}+\frac{x_{0}}{\sigma_{0}^{2}}\right] /\left[\frac{1}{\sigma_{A}^{2}}+\frac{1}{\sigma_{B}^{2}}+\frac{1}{\sigma_{C}^{2}}+\frac{1}{\sigma_{0}^{2}}\right] \text {. }
$$

So fused wind speed based on the Bayesian estimation is $\hat{x}$

$$
\hat{x}=\int_{\Omega} x \frac{1}{\sqrt{2 \pi \sigma_{N}}} \exp \left\{-\frac{1}{2}\left[\frac{x-x_{N}}{\sigma_{N}^{2}}\right]\right\} d x=x_{N} .
$$

The wind speed calculated by Bayesian method is used to predict the wind power by the BPNN relational model between wind speed and wind power.

\section{EXPERIMENTS AND RESULTS ANALYSIS}

In this paper, the relational model between wind speed and wind power is built by a three layer BPNN. The model is trained and testified by historical data of the actual measured wind speed and wind power. Figure 5 shows the actual wind power and calculated wind power by actual wind speed. It illustrates that the accuracy of the model is above $95 \%$ which means that the model is able to describe the relationship between the wind speed and power. The data is obtained from one certain wind farm of China from March 2016 to May 2016.

Figure 6 shows the actual wind speed, forecasted wind speed of NWPA, NWPB, and NWPC and the fused wind speed based on Bayesian method. From Figure 6 we can find that the fused wind speed is more closed to the actual measured wind speed than the three wind speed of NWPA, NWPB, and NWPC. The mean squared errors (MSE) between actual wind speed and the speed forecasting accuracy of the fused wind, wind speed of NWPA, NWPB, and NWPC are 0.84, 1.58, 2.22 , and 0.91 , respectively.

The fused wind speed, wind speed of NWPA, NWPB, and NWPC were used to predict the wind power as the input of the BPNN, respectively. The results were shown in Figure 7 which shows that the MSE between actual wind speed and the speed forecasting accuracy of the fused wind, 
wind speed of NWPA, NWPB, and NWPC are 3.02, 7.13, 4.32, and 4.23, respectively. The predicted power by fused wind speed is more accurate than the original forecasted wind speed of NWPA, NWPB, and NWPC.

The results demonstrate that the proposed Bayesian fusion method can achieve more accurate forecasting wind speed than that forecasted by the original NWPs. By using the fused wind speed, the accuracy of WPP is also much improved.

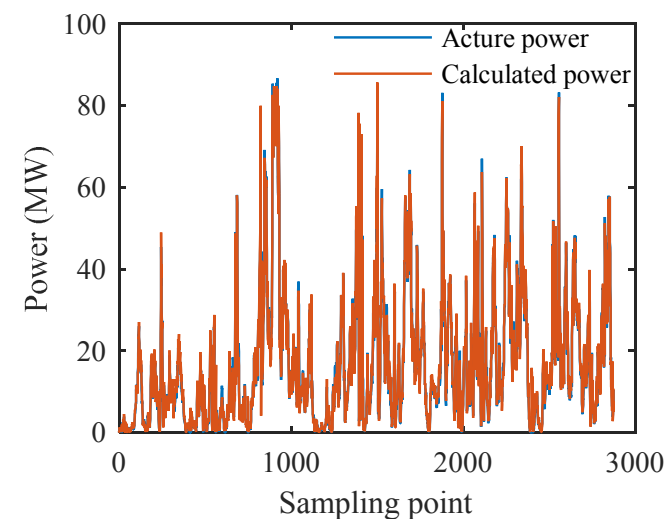

Figure 5. Actual wind power and calculated wind power by actual wind speed based on BPNN

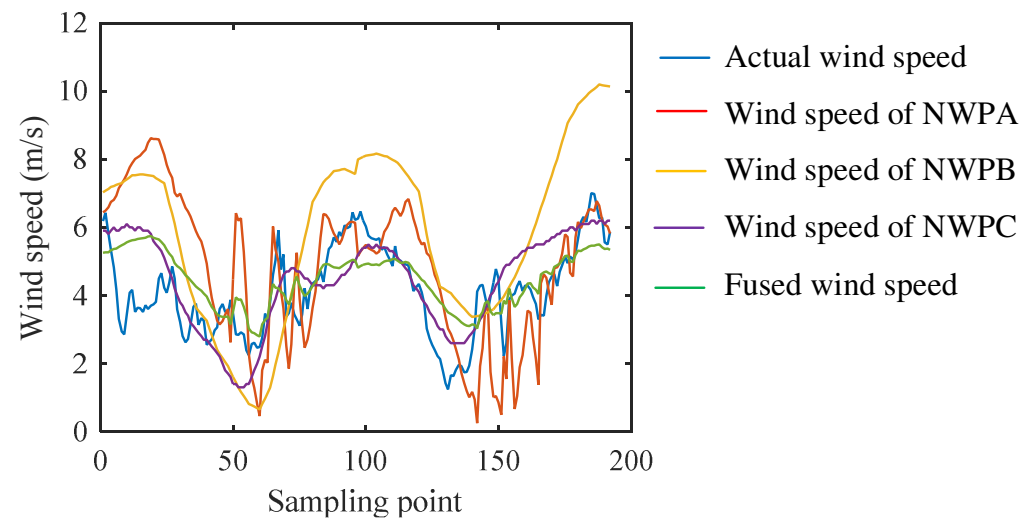

Figure 6. Actual wind speed, fused wind speed, and wind speed of NWPA, NWPB, and NWPC

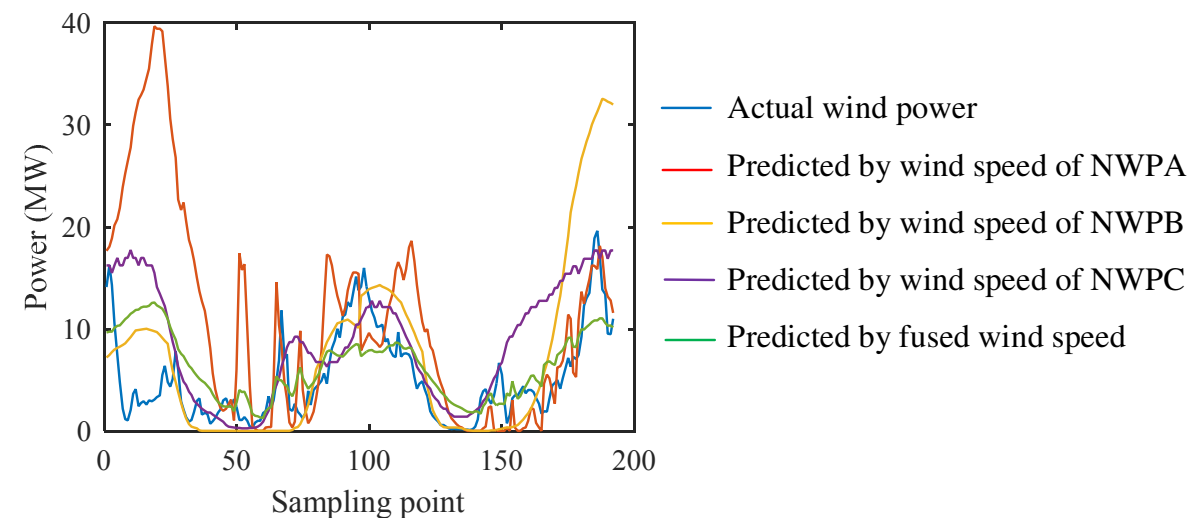

Figure 7. Actual wind power and wind power predicted by fused wind speed, the wind speed of NWPA, NWPB, and NWPC 


\section{CONCLUSION}

This paper presents a new WPP method based on Bayesian method and multi-source NWPs. The proposed method fuses the three NWPs to get more accurate wind speed value; and the fused wind speed is employed to predict the wind power, the accuracy of which can satisfy the demand of the power grid companies. This method has the advantage of lower calculation, higher reliability and practicability.

\section{ACKNOWLEDGEMENTS}

The first author is an overseas researcher under Postdoctoral Fellowship of Japan Society for the Promotion of Science. This work was supported by the Hubei Provincial Natural Science Foundation of China under Grants No. 2016CFB480 and No. 2015CFA010, National Natural Science Foundation of China under Grants No. 61333002 and No. 61203017, and the Foundation Research Founds for China University of Geosciences under Grant No. 2015349120.

\section{REFERENCES}

[1] Rui Pinto. Botterud, Vladimiro Miranda, \& Jean Sumaili, (2011) "Wind power forecasting uncertainty and unit commitment", Applied Energy, vol.88, pp. 4014-4023.

[2] Jae-kun Lyu, J. Jae-Haeng Heo, Mun-Kyeom Kim, \& Jong Keun Park, (2013) "Impacts of wind power integration on generation dispatch in power systems", Journal of Electrical Engineering \& Technology, vol.8, pp. 453-463.

[3] Ch. Ulam-Orgil, Hye-Won Lee, \& Yong-Cheol Kang, (2012) "Evaluation of the wind power penetration limit and wind energy penetration in the mongolian central power system", Journal of Electrical Engineering \& Technology, vol.7, pp. 852-858.

[4] Saurabh S. Soman, Hamidreza Zareipour, Om Malik, \& Paras Mandal, (2010) "A review of wind power and wind speed forecasting methods with different time horizons", Proceedings of 2010 North American Power Symposium, pp. 1-8.

[5] G. Xydis, C. Koroneos, \& M. Loizidou, (2009) "Exergy analysis in a wind speed prognostic model as a wind farm sitting selection tool: A case study in Southern Greece", Applied Energy, vol.86, pp. 2411-2420.

[6] Colm Lowery \& Mark O'Malley, (2012) "Impact of wind forecast error statistics upon unit commitment”, IEEE Transactions on Sustainable Energy, vol.3, pp. 760-768.

[7] Ramesh Babu. N \& P. Arulmozhivarman. P, (2013) "Improving forecast accuracy of wind speed using wavelet transform and neural networks", Journal of Electrical Engineering \& Technology, vol. 8, pp. 559-563.

[8] Xiaojuan Han, Xilin Zhang, Fang Chen \& Zhihui Song, (2012) "Short-term wind speed prediction method based on time series combined with LS-SVM", Proceedings of the 31st Chinese Control Conference, pp. 7593-7597.

[9] Andrew Kusiak, Haiyang Zheng, \& d Zhe Song, (2009) "Short-term prediction of wind farm power:a data mining approach", IEEE Transactions on Energy Conversion, vol.24, pp.125-136.

[10] Wendan Zhang, Fang Liu, Xiaolei Zheng, \& Yong Li, (2015) "A hybrid EMD-SVM based short-term wind power forecasting model”, Proceedings of 2015 IEEE PES Asia-Pacific Power and Energy Engineering Conference, pp. 1-5. 
[11] GUO Pengfei, QI Zhiyuan, \& HUANG Wei, (2016) "Short-term wind power prediction based on genetic algorithm to optimize RBF neural network", Proceedings of the 28th Chinese Control and Decision Conference, pp.1220-1223.

[12] Yanfeng Ge. Peng Liang. Liqun Gao, (2015) "A self-adaptive model for wind power prediction", Proceedings of the 27th Chinese Control and Decision Conference, pp. 1165-1169.

[13] Adnan Anwar \& Abdun Naser Mahmood, (2014) "Enhanced estimation of autoregressive wind power prediction model using constriction factor particle swarm optimization", Industrial Electronics and Applications, pp. 1136-1140.

\section{AUTHORS}

Jianqi An is an associate professor of School of Automation, China University of Geosciences, and an overseas researcher (2016.11- 2018.11) in Department of Computer Science, School of Computing, Tokyo Institute of Technology under Postdoctoral Fellowship of Japan Society for the Promotion of Science. He received his B.S. in 2004, M.S. in 2007, and Ph.D. in 2011 from Central South University. His research interests include detection, Modelling, and advanced control methods for complex process and their applications etc. He is the member of the Chinese Association of Automation (CAA). He published more 20 academic papers, applied for 21 patents, published 2 textbooks.

Zhangbing Chen is a postgraduate student of China University of Geosciences. He was born in Hubei, China, 1992. He received his B.S. from the Hubei University of Technology in 2016. His research interests include wind power prediction, information fusion and data mining.

Min Wu received his B.S. and M.S. degrees in engineering from Central South University, Changsha, China, in 1983 and 1986, respectively, and his Ph.D. degree in engineering from the Tokyo Institute of Technology, Tokyo, Japan, in 1999. He was a faculty member of the School of Information Science and Engineering at Central South University from 1986 to 2014, attaining the position of full professor. In 2014, he moved to the China University of Geosciences, Wuhan, China, where he is a professor in the School of Automation. He was a visiting scholar with the Department of Electrical Engineering, Tohoku University, Sendai, Japan, from 1989 to 1990, and a visiting research scholar with the Department of Control and Systems Engineering, Tokyo

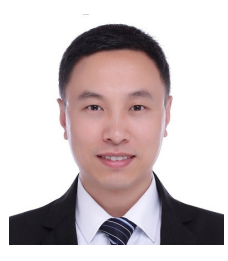
Institute of Technology, from 1996 to 1999. He was a visiting professor at the School of Mechanical, Materials, Manufacturing Engineering and Management, University of Nottingham, Nottingham, UK, from 2001 to 2002. His current research interests include robust control and its applications, process control, and intelligent control.

Dr. $\mathrm{Wu}$ is a member of the Chinese Association of Automation, and a senior member of the IEEE. He received the IFAC Control Engineering Practice Prize Paper Award in 1999 (together with M. Nakano and J. She).

Takao Terano is a professor of Department of Computer Science, School of Computing, Tokyo Institute of Technology. His research interests include genetic algorithm-based machine learning, case-based reasoning, analogical reasoning, distributed artificial intelligence, cooperative agents, computa- tional organization theory, and knowledge dystem development methodology. He is a member of the editorial board of major AIrelated academic societies in Japan and a member of IEEE, ACM, AAAI, and PAAA.

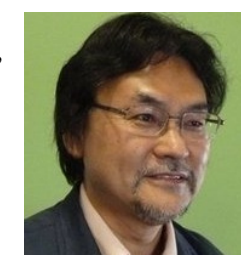


Min Ding is a Lecturer of School of Automation, China University of Geosciences. She received her B.S. from the Central South University in 2009. She had the Ph.D. in Engineering from the Waseda University. Her research interests include renewable energy, control and operation optimization of micro-grid and fault diagnosis of microgrid.

Hua Xie is a postgraduate student of China University of Geosciences. He was born in Shanxi, China, 1992. He received his B.S. from China University of Geosciences in 2015. His research interests include wind power prediction, ormation fusion and data mining.

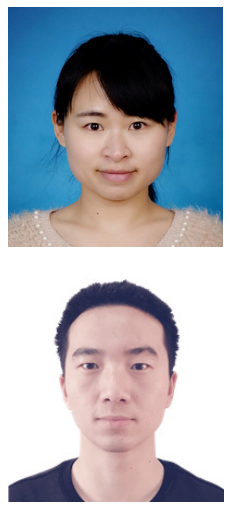

